\title{
The Investigation of a Tertiary Hospital Emergency Service in terms of Quality Standards
}

\author{
Atakan Savrun¹, Emre Gökçen² \\ ${ }^{1}$ Department of Emergency Medicine, Ordu University Faculty of Medicine, Ordu, Turkey \\ ${ }^{2}$ Department of Emergency Medicine, Bozok University Faculty of Medicine, Yozgat, Turkey \\ Received: 01 April 2019, Accepted: 05 April 2020, Published online: 30 April 2020 \\ (C) Ordu University Institute of Health Sciences, Turkey, 2020
}

\begin{abstract}
Objective: Emergency services (ES) are the units where people first apply when they encounter an emergency and provide uninterrupted service 24 hours a day. The quality of these services is as important as the correct diagnosis and treatment of the patients in emergency services. Quality standards for emergency services (QSES) are the scales used for emergency services to provide quality service. In this study, the compliance status of a tertiary hospital emergency service with QSES was investigated.

Methods: Data of emergency service recorded by the quality unit of a tertiary hospital between 01 January 2018 and 31 December 2018 were retrospectively analyzed.

Results: It was found that a total of 90719 patients (27383 in $1^{\text {st }}$ period, 28634 in the $2^{\text {nd }}$ period, 7930 in the $3^{\text {rd }}$ period, 28572 in the $4^{\text {th }}$ period of 2018) applied to the emergency service in 2018 . The patients were kept in the observation room for less than 6 hours, which is the target time for staying of patient. Less than 30 minutes when is the target time of the physician to reach the consultation could not be achieved. Less than $3 \%$ which is the target ratio of the patients re-applying to the emergency service within twenty-four hours with the same complaint was achieved. Less than $1 \%$ which is the target ratio of the patients sent from the emergency service to another center was achieved. Chi-square test test showed that there was a statistically significant difference between the periods in terms of all indicators $(\mathbf{p}<\mathbf{0 . 0 0 1})$.

Conclusion: The hospital's situation regarding QSES was examined and quality targets were mostly met. However, additional measures are required to further improve the quality of the service provided in the emergency service.
\end{abstract}

Key words: Emergency service, quality standards, healthcare

Suggested Citation: Savrun A, Gokcen E..The Investigation of a Tertiary Hospital Emergency Service in terms of Quality Standards. Middle Black Sea Journal of Health Science, 2020; 6(1):129-134.

Address for correspondence/reprints:

Atakan Savrun

Telephone number: +90 (544) 2647070

ORCID-ID 0000-0001-7468-4159

E-mail: atakan4601@ hotmail.com

DOI: $10.19127 /$ mbsjohs.712709

\section{Introduction}

Information about healthcare services can be accessed easily and at any time. Therefore, activity results of healthcare service are reported more clearly and more reliably. In the light of the data obtained, it helps to make business policy and decisions of the organization (Erdem and Ozdagoglu, 2008; Kucuk et al., 2017).

Emergency services (ES) are the units where people first apply when they encounter an emergency and provide uninterrupted service 24 hours a day. Emergency services need to be well organized because it is not known when and how emergencies 
will occur. The quality of these services is as important as the correct diagnosis and treatment of the patients. For this reason, Quality standards for emergency services (QSES) have been developed to provide quality services in emergency services (Altindis ve Unal, 2017; https://Kalite.Saglik.Gov.Tr/Tr,9081/Indikator-

Applemlari.Html). By this way, hospital managers are able to easily identify defective sides of the emergency service of the hospital where they work and take the necessary measures quickly.

In this study, the compliance status of a tertiary hospital emergency service with QSES was investigated

\section{Methods}

\section{Study Design}

This study is a retrospective study and its universe consists of emergency service data recorded by the quality unit of a tertiary hospital between 01 January 2018 and 31 December 2018. The study started after the approval no: 2020/20 by Ethics Committee of Ordu University.

While analyzing the study data, the data collection stages were grouped as follows:

Because the quality unit of the hospital collected the data quarterly, the data were analyzed quarterly in four different periods: 1st period covering JanuaryFebruary-March, 2nd period covering April-MayJune, 3rd period covering July-August-September and 4th period covering October-NovemberDecember.

In the study, the forms named as "The duration of hospitalization of the patients hospitalized in the emergency service", "The rates of patients reapplying to the emergency service within twenty-four hours with the same complaint", "The duration of consultant physician's access to the emergency service", "Patient referral rate of the emergency service" recorded by the quality units of the hospital within the framework of QSES were analyzed retrospectively. According to this;

1. Under the title of "The duration of hospitalization of the patients hospitalized in the emergency service", the total number of patients hospitalized in the observation room, total stay duration (as minutes) of the patients hospitalized in the observation room, the number of the patients hospitalized more than 12 hours among the patients in the observation room and the percentage of patients hospitalized for more than 12 hours among the patients in the observation room were analyzed. As stated in the source information reached, the formula "total of hospitalization durations of the patients hospitalized in the observation/ total number of hospitalizations made in the observation" as the calculation method of stay time in the observation. The duration of the emergency service observation is intended to be under six hours as the target value (QHST, 2020).

2. Under the title of "The rates of patients reapplying to the emergency service within twentyfour hours with the same complaint", the total number of the patients admitted to the emergency service and the number of patients reapplying to the emergency service within twenty-four hours were examined. In line with the source information, the formula "(the number of patients reapplying to the emergency service with the same complaint within 24 hours / the number of patients applying to the emergency service) $\times 100$ " was used as the method of calculating the rates of patients applying to the emergency service again within twenty-four hours with the same complaint and 3\% and below was planned as the target value (QHST, 2020).

3. Under the title of "The duration of consultant physician's access to the emergency service", total number of consultation requests in the emergency service, branches consulted and percentage rates of them, the time in minutes that the physicians coming to the consultation reach the patient, the number of consultations requested during working hours and the number of consultations requested outside working hours were analyzed. Considering the literature, the rate of total time to reach consultation / number of consultation requests to the emergency service in the relevant time period was calculated for the duration of consultant physician's access to the emergency service. The target in this ratio was aimed to be 30 minutes or less (QHST, 2020).

4. Under the title of "Patient referral rate of the emergency service", the total number of patients admitted to the emergency service, the total number of patients referred to another center, the percentage of the patients referred to another center, the percentage of the diagnosis of the patients referred and the referral rates of patients referred to another center were analyzed. In line with the literature source used, the formula "(the number of patients referred to another institution from the emergency service / the number of patients applying to the emergency service)x100 was used to calculate the referral rates in the relevant time interval and the target value was planned to be $\leq 1 \%$ (QHST, 2020). 


\section{Inclusion criteria}

The emergency service forms filled by the quality unit of the hospital between 01 January 2018 and 31 December 2018 were included in the study.

\section{Exclusion criteria}

Incomplete forms were excluded from the study.

\section{Data Analysis}

Frequency analysis of variables was realized, and descriptive statistics were calculated. Whether the frequencies are homogeneously distributed according to the periods was checked by one-way Chi-square test. SPSS v 26 statistics program (IBM Corp., Armonk, NY) was used in calculations.

\section{Results}

A total of 90719 patients, 27383 in 1st period, 28634 in 2nd period, 7930 in the 3 rd period, 28572 in the 4th period, were admitted to the emergency service in 2018.

The data obtained according to the sub-parameters of "The duration of hospitalization of the patients hospitalized in the emergency service" were as follows; it was detected that $23.35 \%(\mathrm{n}=21189)$ of the patients admitted to the emergency service were taken under the observation. In addition, it was found that $1.89(n=1720)$ of the subjects remained in the emergency service for more than 12 hours. The duration of patient stay in the observation room was targeted to be under 6 hours and this target was achieved (Table 1). It was detected that $1.89 \%(\mathrm{n}=$ 1720) of the patients who applied to the emergency service stayed in the emergency service for more than 12 hours (Table 1). Whether the frequencies of the parameters are homogenously distributed according to the periods were checked by one-way Chi-square test. Chi-square test showed a statistically significant difference between periods in terms of all parameters ( $\mathrm{p}<0.001)$. While the highest number of patients hospitalized in the observation room was $40.1 \%(\mathrm{n}=$ $8500)$ in 1 st period, it was determined $1.8 \%(n=383)$ in 4th period as the lowest.

The data obtained according to the sub-parameters of "The rates of patients reapplying to the emergency service within twenty-four hours with the same complaint" were as follows; it was determined that 11929 consultations were requested in the emergency service within a period of one year and this rate was $13.14 \%$ of the total number of patients. It was determined that $3363(3.70 \%)$ of the consultations were requested during working hours while the remaining $1894(2.08 \%)$ were requested outside the working hours. While the target time of the physician to reach the consultation was $<30$ minutes, this period was determined as $>30$ minutes in our hospital, and it was observed that the target value was not achieved (Table 1). Whether the frequencies of the parameters are homogenously distributed according to the periods were checked by one-way Chi-square test. Chi-square test showed a statistically significant difference between periods in terms of all parameters ( $p<0.001)$. In the emergency service, the highest number of consultation requests was $35.2 \%(\mathrm{n}=$ 4200 ) in 2 nd period while the lowest number of consultations, $10.3 \%(\mathrm{n}=1228)$, was requested in 4 th period.

The data obtained according to the sub-parameters of "The duration of consultant physician's access to the emergency service" were as follows; the number and rates of the patients re-admitted to the emergency service within twenty-four hours were $1.42 \%(\mathrm{n}=$ $390), 1.36 \%(\mathrm{n}=391), 4.07 \%(\mathrm{n}=323)$ and $0.32 \%(\mathrm{n}$ $=94$ ), respectively. In total, it was observed that it was $1.32 \%(\mathrm{n}=1198)$. The target value was planned as $33 \%$ and the target value was achieved. Whether the frequencies of the parameters are homogenously distributed according to the periods were checked by one-way Chi-square test. Chi-square test showed a statistically significant difference between periods in terms of all parameters $(\mathrm{p}<0.001)$. It was detected that the number of patients re-admitted to the emergency service within 24 hours was $32.6 \%(\mathrm{n}=$ $391)$ in 2 nd period as the highest and $7.8 \%(\mathrm{n}=94)$ in 4th period as the lowest.

The data obtained according to the sub-parameters of "Patient referral rate of the emergency service" were as follows; a total of 90719 patients applied to the emergency service, of which 187 were transferred to another center for different reasons. The percentage of the patients referred from the emergency service was observed as $0.2 \%$ and the target value, $\leq 1 \%$, has been achieved (Table 1 ). Whether the frequencies of the parameters are homogenously distributed according to the periods were checked by one-way Chi-square test. Chi-square test showed a statistically significant difference between periods in terms of all parameters $(p<0.001)$. The total number of patients referred to another center was $58.8 \%(n=110)$ in 4 th period as the highest while the lowest number of patients referred was detected as $5.3 \%(\mathrm{n}=10)$ in $3 \mathrm{rd}$ period. The hospitalization of COPD patients is increasing in 4th period, which is the winter period. As the number of COPD patients with follow-up is high in Ordu and most of them receive inpatient treatment, the bed capacity is not 
sufficient and for it was thought for this reason that the referral rate is increased.

\section{Discussion}

Data of the emergency service was analyzed retrospectively and the status of achieving the target values specified in QSES was investigated. In addition, consultation times in the emergency service, the time period of the consultation, the proportion of the patients staying in the observation room of emergency service, the patients staying for over 12 hours in the observation room, the patients reapplying to the emergency service within 24 hours, and the rate of referral in the emergency service were investigated. In the direction of the resource information reached, the studies carried out so far and quality standards forms of the emergency service have been examined together. In this study, four parameters thought to affect the emergency service workflow were evaluated together.

Of the patients who applied to ES and whose first assessment was made, the duration of the observation in the emergency service, the number of ones with an observation duration of more than 12 hours and the achievement status of the target value were analyzed in the study. $23.35 \%(\mathrm{n}=21189)$ of 90719 cases who applied to the emergency service were taken to the observation room in the emergency service. The durations of the patients' stay in the observation room of the emergency service were 2.4, 6.0, 5.8 and 0.07 hours according to the periods, respectively. While the highest number of patients hospitalized in the observation room was $40.1 \%(n=8500)$ in 1 st period, it was determined $1.8 \%(\mathrm{n}=383)$ in 4 th period as the lowest. Also, it was detected that $1.89 \%(n=1720)$ of the patients who applied to the emergency service stayed in the emergency service for more than 12 hours. Despite this, $98.11 \%$ of all patients were found to be below 6 hours which is the target time. It was reported in the studies conducted in different centers that the duration of the observation has changed. It was reported in a study conducted that a significant portion, $77 \%$, of the patients were concluded in the first 8 hours in AS however the duration of the observation was extended up to 18 hours in the remaining patient group (Ross et al., 2003). In another study conducted in a tertiary university hospital, it was reported that the average duration of observation was 2 days (Dede, 2006). Kilicaslan et al. reported in their study that the average time of observation of the patients in the emergency service was 2 hours (Kilicaslan et al., 2005). In the study conducted by Oktay et al., they reported the average duration of observation as 3.3 hours (Oktay et al., 2003).
According to the report of the American College of Emergency Medicine, it was reported that the average duration of stay in non-serious illnesses or injuries was 1-2 hours while the duration may increase depending on the diagnosis and clinical condition (www.acep.org). Many factors such as clinical diagnosis of the patients, the examination results of the patients, the knowledge and experience of the physician evaluating the patient, emergency bed status affect the duration of the patient's observation in the emergency service. According to the reports of Karatas et al., emergency services with more than 50000 patient applications per year must have at least 30 beds (Karatas and Ciplak, 2007). However, our hospital, which has over 90000 annual patient applications, has 14 beds in the emergency service. For this reason, it is thought that the patients are discharged, hospitalized or referred faster. Moreover, the fact that there is only one emergency medical specialist in each shift in our emergency service indicates that the diagnosis and treatment organizations of the patients are done quickly. Another reason for the short duration of the observation is that there are large health centers in neighborhood of our hospital, and we think that the patients who may take a long time to diagnose go to these centers.

The patients reapplying to the emergency service within 24 hours were examined in the study. It was determined that $1.32 \%(n=1198)$ of 90719 cases who admitted to the emergency service applied to the emergency service again within 24 hours. The target value, $<3 \%$, has been achieved. Erenler et al. reported in their study that 163,951 patients applied to the emergency service annually and $0.73 \%(n=1210)$ of these patients applied to the emergency service again within 24 hours (Erenler et al., 2014). Another study reported that 312255 people applied to the emergency center per year and $3.6 \%(\mathrm{n}=11420)$ of the subjects applied to the emergency service again in 24 hours (Incesu et al., 2016). Yorulmaz et al. reported that they examined 3-year data of their hospital emergency service, a total of 1083553 patients applied to the emergency service and $0.72 \%(\mathrm{n}=$ 7775) of these subjects re-applied to the emergency service within 24 hours (Yorulmaz et al., 2017). This situation is thought to be related to the fact that the doctors working in the emergency service do not provide sufficient information while discharging the patients. It was concluded that the patients with knowledge did not make unnecessary applications to the emergency services because they learned in which case they should apply to the hospital. 
In the study, the rate of requesting consultation in the emergency service was examined as the percentage. It was detected that the consultation was requested for $13.14 \%(\mathrm{n}=11929)$ [ $(11.67 \%$ in $1 \mathrm{st}$ period $(n=3198), 14.66 \%$ in 2 nd period $(n=4200)$, $41.65 \%$ in 3 rd period $(\mathrm{n}=3303), 4.29 \%$ in 4 th period $(\mathrm{n}=1228)]$ of the applicants in 2018. It was found that consultation was requested for $5.78 \%(\mathrm{n}=5257)$ of these cases in total [3.7\% $(\mathrm{n}=3363)$ during working hours (between 08.00-17.00) and 2.08\% (n $=1894)$ outside working hours (17.00-08.00)]. Consultation is an important practice in patient management in the emergency room (Ozyurt et al.,2018). In various studies conducted in tertiary hospitals in our country, it has been reported that the rates of requesting consultation in ESs were different. Ay et al. reported that they requested consultation for $29.12 \%$ of the subjects in their study including 3609 emergency service applications (Ay et al., 2010). Aydin et al. stated in their study involving 3000 patients that they requested consultations for $39.1 \%$ of the patients (Aydin et al., 2010) while it was reported in another study that 9294 patients were included in the study and $21.6 \%$ of them were requested for consultation (Domez et al., 2017). At least one emergency medical specialist doctor is employed in the emergency service of our hospital while practitioners and physicians with less experience were working in the emergency services in the past. We believe that this rate has decreased since they can terminate most of the complicated patients themselves thanks to the high level of experience and knowledge of emergency medicine specialists. In our study, the mean time to reach the patient (in minutes) of the consultant physician was detected as 30.4, 29.7, 27.5 and 547.3, respectively, and the target value, $<30$ minutes, could not be achieved mostly or could be achieved at the border. We believe that this situation depends on the consulted physicians simultaneously looking at the consultation outside the emergency service, providing the outpatient service and closing the consultation from the system late due to the workload even if the patient was evaluated at the desired time.

The number of patients referred to another center after applying to the hospital emergency service was examined in our study. It was observed that the rates of the subjects referred to the center other than the emergency service to the total subjects applied were $0.09 \%(\mathrm{n}=26), 0.14 \%(\mathrm{n}=41), 0.12 \%(\mathrm{n}=10)$ and $0.38 \%(\mathrm{n}=187)$, respectively, and the rate was $0.20 \%$ $(\mathrm{n}=187)$ in total. The target value, < $1 \%$, was achieved. The total number of patients referred to another center was $58.8 \%(\mathrm{n}=110)$ in 4 th period as the highest while the lowest number of patients referred was detected as 5.3\% $(\mathrm{n}=10)$ in 3rd period. The hospitalization of COPD patients is increasing in 4th period, which is the winter period. As the number of COPD patients with follow-up is high in Ordu and most of them receive inpatient treatment, the bed capacity is not sufficient and for it was thought for this reason that the referral rate is increased. In the light of the source information available, no research was found in the literature regarding the patients referred from the emergency service. The study shows that the emergency service is successful in referrals. This situation is thought to be related to the fact that technical facilities of the emergency services are good and emergency medicine specialists can solve most patients' problems at their centers thanks to their knowledge, skills and experience.

\section{Conclusion}

The situation of the hospital emergency service was analyzed with respect to quality standards and it was determined that the quality targets were mostly achieved. However, I believe that the recommendations given below should be followed to further improve the quality of the service provided in the emergency service.

1. The number of emergency medical specialists should be increased in all centers.

2. We believe that work planning should be made so that there is at least one emergency medicine specialist at each shift in the centers with adequate emergency medicine specialists

3. Emergency medicine specialists should be assigned in the centers with high technical equipment and infrastructure.

4. More extensive studies evaluating QSES should be done and preventive measures should be taken for the deficiencies observed.

Ethics Committee Approval: The Ordu University Clinical Research Ethics Committee approved the protocol of this study (KAEK 2020-20).

Peer-review: Externally peer-reviewed.

Author Contributions: Concept- A.S.; Design-A.S, E.G.; Materials- A.S; Data Collection and/or Processing- A.S, E.G..; Literature Review- A.S, E.G.; Writing- A.S, E.G..; Critical Review- A.S.

Conflict of Interest: No conflict of interest was declared by the authors.

Financial Disclosure: The authors declared that this study hasn't received no financial support. 


\section{References}

Altındis S, Unal O. Situation of Turkey About Emergency Service Quality Standards. Journal of Biotechnology and Strategic Health Research 2017; 2: 51-59.

Ay D, Akkas M, Sivri B. Patient population and factors determining length of stay in adult ED of Turkish University Medical Center. J Emerg Med. 2010 Mar; 28 (3): 325-30.

Aydin T, Aydin S A, Koksal O, Ozdemir F, Kulac S, Bulut M. Evaluation of Features of Patients Attending the Emergency Department of Uludag University Medicine Faculty Hospital and Emergency Department Practices. JAEM 2010: 163-8

Donmez S S, Durak V A, Torun G, Koksal O, Aydın S. Analysis of the process of consultations in the emergency department. Journal of Uludag University Faculty of Medicine 43 (1) 23-28, 2017

Dede F. Epidemiological Examination of Patients Aged 65 and Over Admitted to Hacettepe University Adult Emergency Clinic Between January 2005 and December 2005. Specialization thesis. Hacettepe University, Faculty of Medicine. 2006.

Erenler AK, Akbulut S, Guzel M, Cetinkaya H, Karaca A, Turkoz B, Baydin A. Causes of Excessive Crowd in the Emergency Department: Experiences and Recommendations of an Education Research Hospital. Turkey Emergency Medicine Journal 2014; 14(2): 59-63.

Emergency Department Waiting Times: American College of Emergency Medicine. Available from: URL: www.acep.org Fact Sheets. (access: 26.04.2020).

Erdem S, Ozdagoglu G. Analysis of Emergency Patient Data with Data Mining of a Research and Application Hospital in the Aegean Region. Anadolu University Journal of Science and Technology 2008; 9(2): 261-270.

Health, quality, accreditation and employee rights department. Ministry of Health website. Available from:

URL: https://Kalite.Saglik.Gov.Tr/Tr,9081/IndikatorApplemlari.Html (Download Date: 20.03.2020).

Incesu E, Beylık U, Kucukkendırcı H. The problem of re-admission to emergency services: a case study for a state hospital emergency service in Turkey. Academic Overview Journal 2016; 5381: $1-13$

Kucuk KM, Bas U. A Field Study on The Importance of Computerized Bookkeeping and Benefits of Computerized Accounting Softwares.
International Journal of Economic Studies 2017;3(2):131-147.

Karatas Ciplak S. Emergency service architecture (Doctoral dissertation) Izmir, Dokuz Eylul University, 2007.

Kilicaslan I, Bozan H, Oktay C, Goksu E. Demographic properties of patients presenting to the emergency department in Turkey. Turkey Journal of Emergency Medicine 2005; 5(1): 5-13.

Oktay C, Cete Y, Eray O, Pekdemir M, Gunerli A. Appropriateness of Emergency Department Visits İn a Turkish University Hospital. Croatian Medical Journal 2003; 44(5): 585-91

Ozyurt E , Aykutlug M , Ugurlu D , Kudas S M , Ilican B , Cimen G, Alper M. SMS application in emergency service consultation Diskap1 Hospital model. Health Care Acad J 2018 :5 (4); 275-81

Ross MA, Compton S, Richardson D, Jones R, Nittis T, Wilson A. The UseAndEffectiveness of An Emergency Department Observation Unit For Elderly Patients. Annals of Emergency Medicine 2003; 41(5): 668-77.

Quality Health System in Turkey. (QHST),2020; https://kalite.saglik.gov.tr/TR,8785/turkiyesaglikta-kalite-sistemi.html (access: 26.04.2020).

Yorulmaz M, Karaalp M, Bukecik N, Ozyilmaz A F. Evaluation of Emergency Service Again Application Rating. Journal of Social and Technical Researches 14, 2017;14, 92-99). 